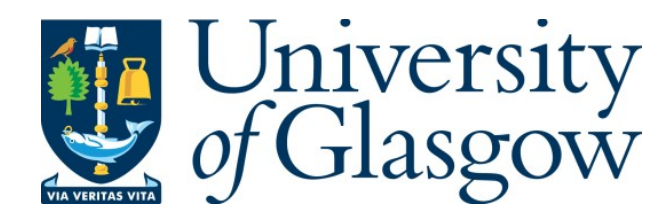

Jacobs, K. J.P., Baba, R., Stevens, B. J., Mukai, T., Ohnishi, D., and Hogg, R. A. (2016) Characterisation of High Current Density Resonant Tunneling Diodes for THz Emission Using Photoluminescence Spectroscopy. In: SPIE Photonics West, San Francisco, CA, USA, 13-18 Feb 2016, (doi:10.1117/12.2212669).

There may be differences between this version and the published version. You are advised to consult the publisher's version if you wish to cite from it.

http://eprints.gla.ac.uk/119898/

Deposited on: 7 November 2016

Enlighten - Research publications by members of the University of Glasgow http://eprints.gla.ac.uk 


\title{
Characterisation of High Current Density Resonant Tunnelling Diodes for THz Emission Using Photoluminescence Spectroscopy
}

\author{
Kristof J. P. Jacobs ${ }^{a}$, Razvan Baba ${ }^{b}$, Benjamin J. Stevens ${ }^{a}$, \\ Toshikazu Mukaic, Dai Ohnishic ${ }^{\mathrm{c}}$, Richard A. Hogg ${ }^{\mathrm{b}}$ \\ ${ }^{a}$ Department of Electronic \& Electrical Engineering, University of Sheffield, \\ Centre for Nanoscience \& Technology, North Campus, Broad Lane, Sheffield, S3 7HQ, United \\ Kingdom; ' School of Engineering, University of Glasgow, Rankine Building, Glasgow, G12 8LT, \\ United Kingdom; 'Optical Device Research and Development Div., Sensor Business Strategy, \\ Rohm Co. Ltd.,21 Saiin Mizosaki-cho, Ukyo-ku, Kyoto 615-8585, Japan
}

\begin{abstract}
Resonant tunneling diodes (RTDs) provide high speed current oscillation which is applicable to THz generation when coupled to a suitably designed antenna. For this purpose, the InGaAs/AlAs/InP materials have been used, as this system offers high electron mobility, suitable band-offsets, and low resistance contacts. However for high current density operation $\left(\sim \mathrm{MA} / \mathrm{cm}^{2}\right)$ the epitaxial structure is challenging to characterize using conventional techniques as it consists of a single, very thin AlAs/InGaAs quantum well (QW). Here, we present a detailed low temperature photoluminescence spectroscopic study of high current density RTDs that allow the non-destructive mapping of a range of critical parameters for the device. We show how the doping level of the emitter/collector and contact layers in the RTD structure can be measured using the Moss-Burstein effect. For the full device structure, we show how emission from the QW may be identified, and detail how the emission changes with differing indium composition and well widths. We show that by studying nominally identical, un-doped structures, a type-II QW emission is observed, and explain the origin of the type-I emission in doped devices. This observation opens the way for a new characterization scheme where a "dummy" RTD active element is incorporated below the real RTD structure. This structure allows significantly greater control in the epitaxial process.
\end{abstract}

Keywords: Resonant tunnelling diode, quantum well, optical spectroscopy, terahertz

\section{INTRODUCTION}

Resonant tunnelling diodes (RTDs) find application in terahertz wave electronics and photonics including spectroscopic sensing, imaging, and ultra-broadband wireless communications [1]. The RTD is a compact, coherent, room temperature $\mathrm{THz}$ device that lends itself to integration with other functional electron/optical devices [2]. As wireless data rates have been doubling every 18 months, following Edholm's law of bandwidth, it is predicted that higher carrier frequencies will need to be accessed for future data rates, and presently unused regions of the electromagnetic spectrum will need to be utilised [3]. To date, the resonant tunnelling diode (RTD) is recognised as the fastest electronic device, with a reported fundamental oscillation at $1.86 \mathrm{THz}$ [4]. The majority of THz RTDs are based upon the InGaAs/AlAs/InP material system for high frequency performance. Fig. 1(a) shows a typical I-V characteristic of a dual pass high current density (J 700 kA/ $\mathrm{cm}^{2}$ ) InGaAs/AlAs/InP RTD [5] and (b) pictographically shows a typical monolithically integrated RTD THz emitter [6]. THz radiation is generated when the RTD biased in the negative differential conductance (NDC) region and a standing wave of the electromagnetic field is formed within the slot antenna. The RTD THz device requires tight growth tolerances including layer thickness, alloy composition, and doping concentration to achieve large voltage spans $(\Delta \mathrm{V})$ and current spans $(\Delta \mathrm{I})$, as depicted in Fig. 1(a), for a high output power. The maximum oscillation frequency is limited by the transit time of the charge carriers in the NDC region, as illustrated in Fig. 1(a). Good wafer-to-wafer reproducibility and wafer-scale uniformity are required for the low cost mass-manufacture of these devices. So far, the required level of reproducibility of the RTD for high-volume low cost manufacture (typically less than $5 \%$ of a prespecified mean for electronic device characteristics [7]) has not been fully demonstrated yet. 

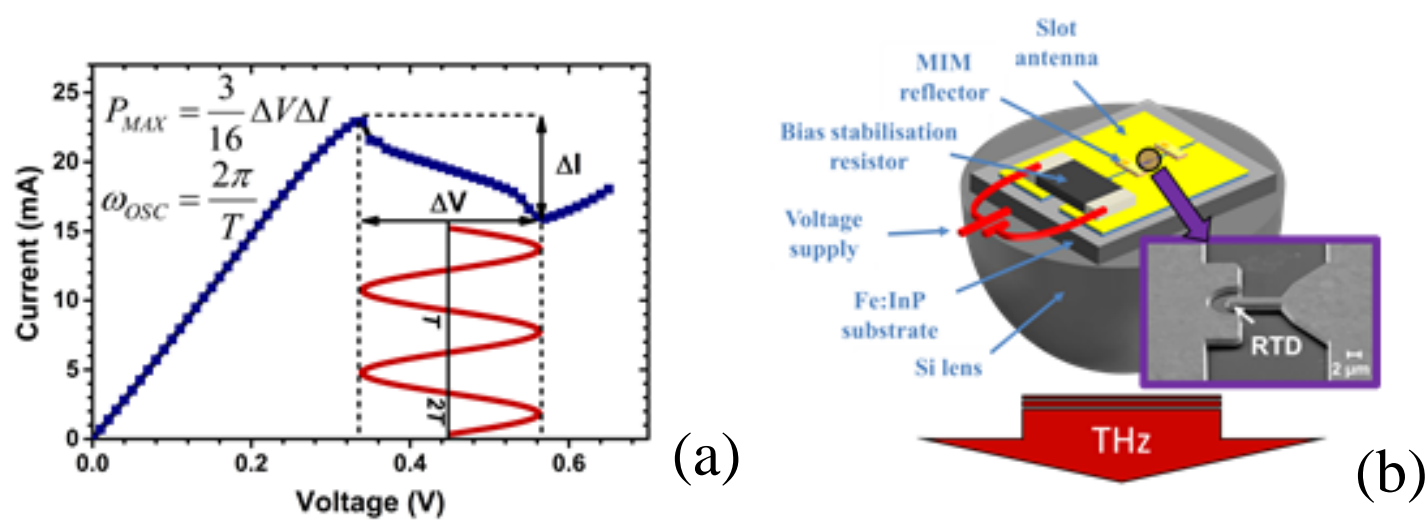

Fig.1. (a) I-V characteristic of a typical $700 \mathrm{kA} / \mathrm{cm}^{2} \mathrm{RTD}$ (b) Schematic of a typical RTD THz emitter. The SEM image is zoomed-in on the RTD and slot antenna.

The crystal growth and device fabrication of these devices is challenging as the current-voltage (I-V) characteristic and carrier transport of the RTD are highly sensitive to the epitaxial layers parameters (i.e. thickness, composition, and doping) and final device dimensions [8]. The characterisation of the structural parameters of the RTD required for growth optimisation is problematic as the structure is typically very thin $(<10 \mathrm{~nm})$ and is not periodic. Transmission electron microscopy (TEM), secondary ion mass spectrometry (SIMS), electrochemical capacitance-voltage (eCV) profiling [9], X-ray diffractometry (XRD), and room temperature photoluminescence (PL) spectroscopy are typical characterisation techniques for the material analysis of epitaxial structures, yet they provide either limited information in a short cycle-time (eCV, XRD, PL), or are costly and have a long cycle-time with regard to growth optimisation (e.g. TEM, SIMS). XRD and PL are regarded as highly favorable production environment characterisation tools as they are non-destructive. To engineer higher current density/frequency devices, a precise knowledge of the resonant energy levels is also important. In particular, the energy of the first confined resonant electronic state above the conductionband of the emitter, $\Delta \mathrm{E}$, is a critical parameter. Determining the absolute energetic position of the resonant energy level using conventional characterisation techniques has not been possible so far.

In this paper, we report on low temperature PL spectroscopy of $\sim \mathrm{MA} / \mathrm{cm}^{2}$ resonant tunnelling diodes (RTDs) for rapid and non-destructive characterisation of the growth process and device optimisation. We show how to apply PL to noninvasively measure the doping concentration of the emitter/collector and contact layers by using the Moss-Burstein (MB) shift. This measurement lends itself readily to wafer mapping. We go on to demonstrate how the emission from the individual layers may be investigated in detail using selective layer excitation by scanning the excitation laser across the edge of the sample. Furthermore, we propose and demonstrate a novel characterisation technique for the full device structure using PL through a combination of type-I and type-II emission using a 'dummy' RTD buried in the RTD structure. This technique allows important structural parameters such as alloy content and QW thickness, interface roughness and alloy scattering to be investigated, and the absolute energy of the first electron resonant energy level of the RTD to be determined.

\section{PHOTOLUMINESCENCE SPECTROSCOPY OF RESONANT TUNNELLING DIODE}

Fig. 2(a) shows a low temperature PL spectrum at $15 \mathrm{~K}$ of a typical RTD structure with a $4.5 \mathrm{~nm}$ compressively strained In0.80Ga0.20As quantum well (QW) sandwiched between two $1.1 \mathrm{~nm}$ tensile strained AlAs barriers and highly doped $(\mathrm{n}=3 \times 1018 \mathrm{~cm}-3)$ lattice matched InGaAs for the emitter and collector layers. A frequency doubled neodymium doped yttrium vanadium oxide (Nd:YVO4) laser at $532 \mathrm{~nm}$ was used as excitation source. The luminescence was dispersed by a double grating Bentham DMc150 monochromator and detected by an InGaAs transimpedance amplified photodetector. The lower left inset of Fig. 2 shows the identical spectrum plotted on a linear scale. Three distinctive emission features are observed at $789 \mathrm{meV}, 834 \mathrm{meV}$, and a broad emission at $\sim 920 \mathrm{meV}$. We previously attributed these emission lines to the undoped InGaAs, QW, and the $\mathrm{n}+$ InGaAs layers, respectively [10]. We highlight the need for low temperature measurements as raising the sample temperature above $50 \mathrm{~K}$ results in a broadening and merging of these features. We also identified that the PL emission energy from the $n+I n G a A s$ layer increases with doping concentration due to the MB effect. Using the MB shift, we have been able to non-destructively measure the free-electron concentration of the emitter/collector and contact layers using low temperature PL and map the uniformity across full 2" wafers. The top right 
inset of Fig. 2(b) shows the calibration curve, measured on a $n+\operatorname{InGaAs}$ calibration test-structure, to relate the $n+$ InGaAs emission energy to a free-electron concentration in that layer. More details of this calibration are reported elsewhere [10]. To minimise the PL from the undoped InGaAs and QW layers interfering with the measurement, the emission is measured at $-20 \mathrm{~dB}$ intensity at the high energy doping tail. Arrows in Fig. 2(a) indicate the $-20 \mathrm{~dB}$ energies at the high energy tails of the emitter/collector and contact layers. Using the doping calibration curve, free-electron concentrations of $6 \times 1018 \mathrm{~cm}-3$ and $3 \times 1019 \mathrm{~cm}-3$ are determined for the emitter/collector and contact layers, respectively. We have confirmed good agreement between the measured free-electron concentrations with PL and measurements carried out using eCV profiling.

To investigate the emission lines of the QW and InGaAs layers in greater detail, selective layer excitation was applied by using a PL linescan technique across the edge of the wafer. Fig. 2(b) shows the measured PL intensity as a function of energy for 103 scans measured across the edge of the sample in the epitaxial direction with a 10 micron step size. The technique, schematically illustrated in the inset of Fig. 2(b), allows a partially quantitative PL map with higher resolution than can be obtained using standard positioning of the stages due to the very shallow angle used (less than 1 degree). Due to the finite excitation spot size, the PL emission from the $4.5 \mathrm{~nm}$ QW is spread across multiple spectra. This technique also allows a more accurate measurement of the emission linewidths as the spectral overlap between emission lines is minimised. PL linewidths of $7 \mathrm{meV}$ and $27 \mathrm{meV}$ are measured for the undoped bulk InGaAs and QW emission lines, respectively. For the undoped bulk InGaAs emission linewidth, variations in alloy composition of $<1 \%$ are estimated over the exciton volume. The energetic position of the InGaAs emission line also confirms lattice matched growth on InP. Whilst the energetic position of the InGaAs emission line provides information of the ternary alloy composition, the energetic position of the QW emission provides information of the average well width, and its linewidth provides a good measure of the structural perfection. As the indium concentration in the QW approaches the binary compound InAs, alloy broadening in the QW is expected to be less pronounced than in the case of the bulk alloy. Within the exciton volume, we therefore expect alloy compositional fluctuations of less than $0.5 \%$ in the In0.80Ga0.20As QW. Low alloy broadening is required for good device performance to minimise alloy scattering which degrades device performance. With regard to QW interface roughness, no evidence is found in Fig. 2(b) of extended ML fluctuations due the lack of strong discrete luminescence peaks in the spectra. The lateral extension of interface roughness is therefore expected to be less than the exciton size (few tens of $\mathrm{nm}$ for the in-plane direction [11]) and the excitons are directly probing the interface roughness of the barrier interfaces [12]. We note that ML fluctuations of the barrier thickness are also expected to have a significant role in modifying the linewidth of the transition.

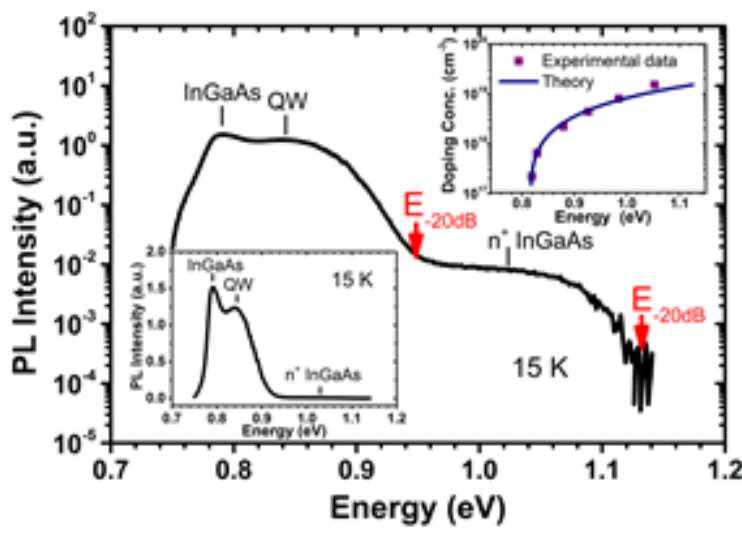

(a)

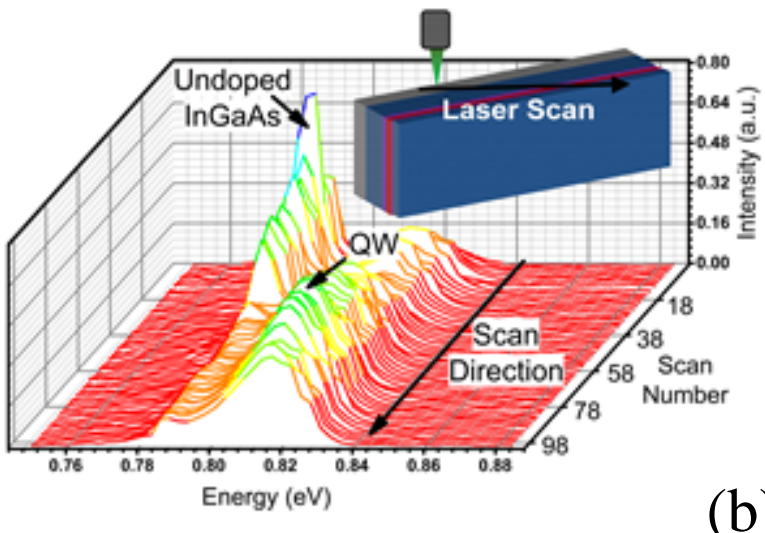

Fig.2 (a). Low temperature PL spectrum of the RTD structure on a logarithmic scale. The lower left inset shows the identical spectrum plotted on a linear scale. The top right inset shows the PL calibration curve for doping measurements. (b) PL measured at $15 \mathrm{~K}$ as a function of energy measured along across the edge of the sample. The inset pictographically shows the PL line scan technique across the edge of the sample.

\section{PHOTOLUMINESCENCE CHARACTERISATION FOR RESONANT TUNNELLING DIODE OPTIMISATION}

The energetic position and linewidth of the QW emission in Fig. 2 provide limited information about the structural parameters of the RTD. A type-I QW luminescence characteristic is observed in RTD structure as the doping induced electron density within the QW is high $(1018 \mathrm{~cm}-3)$ which may recombine with captured photo-generated holes in the 
well [13]. For this type-I recombination, low temperature PL provides a measure of the QW width and composition. A unique, independent measurement of these two parameters is not possible, nor is the absolute energetic position of the QW resonance known. This would be possible if both type-I and type-II emission from the QW were observed. This combination of type-I and type-II emission is therefore expected to provide more information of the structural composition and electronic band structure of the RTD.

To enable type-I and type-II QW emission from the RTD, a structure was designed and grown with an identical undoped 'dummy' RTD buried in the InGaAs buffer layer below the active QW. Fig. 3(a) shows a schematic of the layer structure. In order to accurately deduce the structural parameters from the THz RTD, we highlight the need to grow both QWs in a single structure to ensure that identical growth conditions are applied to both QWs.

\begin{tabular}{|c|c|c|c|c|}
\hline $8 \mathrm{~nm}$ & $\ln _{0 x} C_{n_{0}}$ As: & $n=2 \times 10^{71} \mathrm{~cm}^{3}$ & colloctor contast layer & \multirow{10}{*}{$\begin{array}{c}\text { Active } \\
\text { RTD }\end{array}$} \\
\hline $15 \mathrm{~nm}$ & 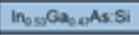 & $n=2 \times 10^{-17} \mathrm{~cm}^{3}$ & colloctor contast layer & \\
\hline $25 \mathrm{~nm}$ & Inosgane $A_{2}$ Si & $n=3 \times 10^{* 14} \mathrm{~cm}^{3}$ & collector & \\
\hline $20 \mathrm{~nm}$ & Inossas ons & undoped & spacer & \\
\hline $1.1 \mathrm{~nm}$ & Alas & undoped & barrier & \\
\hline $4.5 \mathrm{~nm}$ & 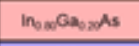 & undoped & $a w$ & \\
\hline $1.1 \mathrm{~nm}$ & Als & undoped & barrier & \\
\hline $2 \mathrm{~nm}$ & 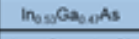 & undoped & spacer & \\
\hline $20 \mathrm{~nm}$ & Inosganous:Si & $n=3 \times 10^{16} \mathrm{~cm}^{3}$ & emitter & \\
\hline $400 \mathrm{~nm}$ & Inossan as: & $n=2 \times 10^{-11} \mathrm{~cm}^{3}$ & emitter contact layer & \\
\hline $100 \mathrm{~nm}$ & $\ln _{0,0} G_{\text {ane }}$ As & undoped & Buffer layer & \multirow{5}{*}{$\begin{array}{c}\text { Dummy } \\
\text { RTD }\end{array}$} \\
\hline $1.1 \mathrm{~nm}$ & Alas & undoped & bamier & \\
\hline $4.5 \mathrm{~nm}$ & Inowatents & undeped & $a w$ & \\
\hline $1.1 \mathrm{~nm}$ & $\ln _{0 \times} G_{2} x_{2} A_{s}$ & undoped & barrier & \\
\hline $100 \mathrm{~nm}$ & Inosonous & undeped & buffer layer & \\
\hline \multicolumn{4}{|c|}{ Semiinsulating InP substrate (350 um) } & \\
\hline
\end{tabular}

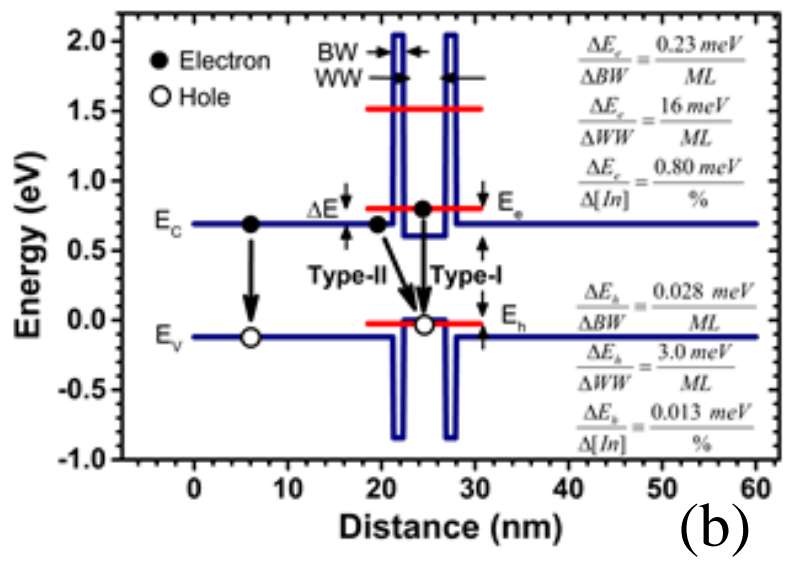

Fig.3 (a) schematic of the RTD layer structure with 'dummy' RTD buried in the InGaAs buffer layer (b) Band-structure at $15 \mathrm{~K}$ of a InGaAs/AlAs/InP RTD with undoped emitter/collector layers showing the possible energetic transitions. The horizontal red lines indicate the first and second electron states and first heavy-hole state. ML, BW, WW, and [In] are defined as monolayer, barrier width, well width, and indium concentration of the QW, respectively.

A sensitivity analysis was carried out to investigate the effect of the layer thickness of the AlAs barriers, the thickness of the compressively strained quantum well (QW), and the indium content of this nominally $\operatorname{In}_{0.80} \mathrm{Ga}_{0.20} \mathrm{As} \mathrm{QW}$ on the confinement energy of the first electron and heavy-hole states. Fig. 3(b) shows the low temperature band-structure of the undoped RTD structure with the resonant energy levels of the RTD structure modelled using a transfer matrix method, and band alignments determined by the model-solid theory. The possible luminescence transitions which may occur in such a structure are indicated by arrows. The effect of the structural parameters is included in the figure. From our calculations, we confirm that the confinement energy of the electrons in the QW is a strong function of the quantum well width (WW) and the indium content [In], but is significantly less sensitive to variations in barrier width (BW). For the holes, as expected for a significant increase in mass (from $0.03 \mathrm{~m} 0$ to $0.60 \mathrm{~m} 0$ ), there is a considerable reduction in the sensitivity of the confinement energy to all parameters. Type-II emission is expected to occur from the 'dummy' RTD as the electrons in the spacer layers will recombine with confined holes in the QW as the electron escape time of the photogenerated electrons ( $\sim 100 \mathrm{fs}$ ) is significantly less than the free exciton-recombination time ( $\sim 1 \mathrm{~ns})$. To a first approximation, the energy of the type-II transition, as indicated in Fig. 3(b), is dominated by the alloy composition of the QW and band-offsets, whilst the energy of the type-I transition, is dominated by the well thickness and alloy composition (once the band-offsets have been determined). Low temperature PL measurements were carried out at $15 \mathrm{~K}$ on the RTD structure shown in Fig. 3(a).

Fig. 4 shows power dependent low temperature PL spectra measured at $15 \mathrm{~K}$ on (a) the as-grown sample, and (b) a sample with the active QW selectively etched off. We etched the active QW in Fig. 4(b) to prevent spectral overlap which would interfere with the measurement. In addition to the emission features at $803 \mathrm{meV}$ and $829 \mathrm{meV}$ as previously observed in Fig.2(a), weak emission is observed below the band-gap energy of bulk InGaAs at 769 meV (lowest excitation power density). This emission lends evidence to the type-II QW emission, as the emission energy is below the band-gap energy of ternary InGaAs and shows a strong power dependency, as expected for type-II emission [14]. The energetic position of the type-II QW emission provides information on the depth of the potential well, due to the low quantisation energy in the heavy-hole band. For a four order magnitude increase in excitation power density, a 
linewidth broadening from $8 \mathrm{meV}$ to $17 \mathrm{meV}$ is observed for the type-II emission. This is attributed to the electrostatically induced triangular QW which becomes more pronounced with increasing excitation power density due to the Coulomb attraction between the electrons and trapped holes. This interaction broadens the electronic states and the effect of ML barrier fluctuations are therefore expected to become more significant due to the increased electron wave function overlap with the barriers. Furthermore, due to the asymmetric doping profile of the QW, the electrostatic potential formed is expected to be asymmetric, with this asymmetry (and hence broadening) becoming more pronounced with increasing PL excitation power. For the QW hole states contributing to this emission linewidth, lifetime broadening is negligible, and the sensitivity of the emission energy to structural parameters is very weak. If a linewidth of 7 meV is assumed for the electrons in the ternary InGaAs from the measured InGaAs linewidth, then the holes confined within the QW contribute around $4 \mathrm{meV}$ to the linewidth broadening. We note that the type-I QW transition has a total linewidth of $20 \mathrm{meV}$, and that the ratios of these broadenings are similar to the ratio of effective masses of the electrons and holes. Furthermore, whilst the energetic position of type-II QW emission contains information on the alloy composition of the quantum well, type-I QW emission allows the QW thickness to be deduced.
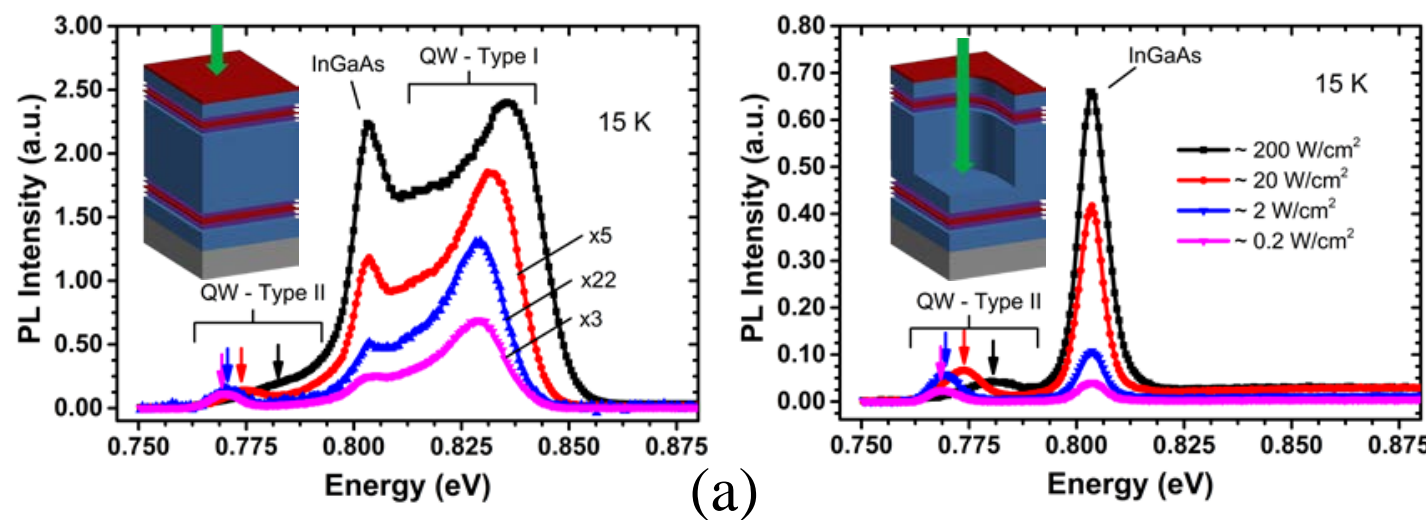

Fig.4. Excitation dependent PL spectra of the RTD structure at $15 \mathrm{~K}$ (a) before and (b) after etching the active QW. The insets pictographically show the etched structures.

Fig. 5 shows the measured peak emission energies of both QWs as a function of the third root of the excitation intensity. The emission line from the 'dummy' QW shows a stronger excitation power density dependence than the active QW, whilst the bulk InGaAs emission does not follow this trend and remains constant.

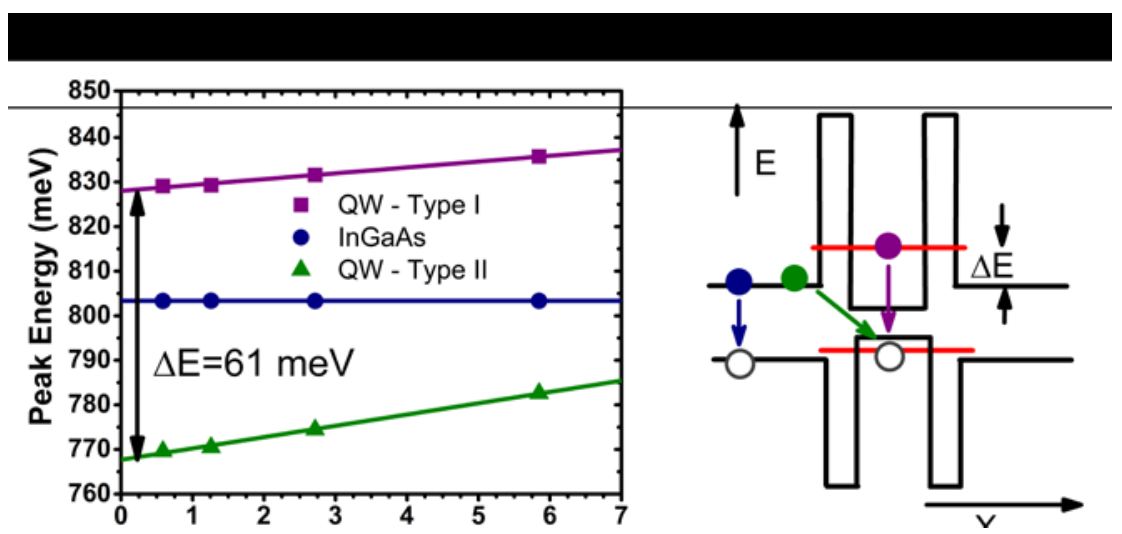

Fig.5. Peak emission energy as a function of excitation density for the active (type-I) and ‘dummy' RTD QW (type-II). The band-structure shows the possible luminescence transitions.

The energetic difference between the type-I and type-II emission provides a direct measurement of the absolute energetic position of the first electronic state of the RTD above the emitter layer energy, which is a critical parameter. To minimise the effect of band-bending due to the photogenerated charge carriers, and of possible heating due to the laser excitation in the analysis, we extrapolated the peak emission energies to zero excitation power density and obtain a $\Delta \mathrm{E}$ value of 61 $\pm 5 \mathrm{meV}$. A band-offset ratio of 67:33 is deduced from these measurements, which is in-line with expected values 
( 76:24 for the InAs/GaAs material system [15]). From theory, we calculated a $\Delta \mathrm{E}$ of $110 \mathrm{meV}$, which is most likely an overestimated value due to band-offset uncertainties. The technique we have proposed and demonstrated is therefore very useful as no prior knowledge of the band-offset of this highly strained heterostructure is required to determine the relative energetic position of the first resonant energy level of the RTD.

\section{CONCLUSIONS}

We have reported on the use of PL characterisation as a rapid, low-cost, and non-destructive tool for growth monitoring and optimisation of high current density InGaAs/AlAs/InP high current density RTD for THz applications. We showed how PL charactrisation may be applied to measure the absolute doping concentration of the highly doped emitter/collector and contact layers using the Moss-Burstein shift. We demonstrated how selective layer excitation may also be applied to investigate emission linewidths through minimising the spectral overlap between emission lines. We designed and grew a new RTD structure with a 'dummy' RTD buried in the InGaAs buffer layer which allows us to monitor the structural composition in detail. With our technique, we were able to measure the relative position of the first electron resonant energy level with regard to the emitter energy, $\Delta \mathrm{E}$, through the analysis of type-I and type-II QW emission.

\section{Acknowledgements}

The authors wish to gratefully acknowledge D. T. D. Childs and Prof. O. Wada for fruitful discussions. K.J.P. Jacobs gratefully acknowledges EPSRC for a PhD studentship.

\section{REFERENCES}

[1] M. Tonouchi “Cutting-edge terahertz technology”, Nature Photon., vol. 1, pp.97 - 105 , 2007.

[2] Romeira B, Figueiredo JML, Ironside CN, Kelly AE, Slight TJ. “Optical control of a resonant tunneling diode microwave-photonic oscillator”. IEEE Photon. Technol. Lett. 2010;22(21):1610.

[3] S. Cherry "Edholm’s law of bandwidth”, IEEE Spectrum, vol. 41, no. 7, pp.58 -60, 2004.

[4] S. Suzuki, M. Asada, "Terahertz oscillators and receivers using electron devices for high-capacity wireless communication", Proceedings of SPIE Vol. 9483, 948309 (2015)

[5] Jacobs, K.J.P.; Stevens, B.J.; Wada, O.; Mukai, T.; Ohnishi, D.; Hogg, R.A., "A Dual-Pass High Current Density Resonant Tunneling Diode for Terahertz Wave Applications," in Electron Device Letters, IEEE , vol.36, no.12, pp.1295-1298, Dec. 2015

[6] Jacobs, K.J.P.; Stevens, B.J.; Wada, O.; Mukai, T.; Ohnishi, D.; Hogg, R.A., "A dual-pass high current density resonant tunnelling diode terahertz emitter," in Infrared, Millimeter, and Terahertz waves (IRMMW-THz), 2015 40th International Conference on , vol., no., pp.1-2, 23-28 Aug. 2015

[7] Missous, M.; Kelly, M.J.; Sexton, J., "Extremely Uniform Tunnel Barriers for Low-Cost Device Manufacture," Electron Device Letters, IEEE , vol.36, no.6, pp.543,545, 2015

[8] H. Sugiyama, A. Teranishi, S. Suzuki, M. Asada, "High-uniformity InP-based resonant tunneling diode wafers with peak current density of over $6 \times 10^{5} \mathrm{~A} / \mathrm{cm}^{2}$ grown by metal-organic vapor-phase epitaxy, Journal of Crystal Growth, vol. 336, no. 1, pp.24-28, 2011

[9] P. Blood, "Capacitance-voltage profiling and the characterisation of III-V semiconductors using electrolyte barriers", Semicond.

Sci. Technol. 1 (1986), 7

[10] K.J.P. Jacobs, B.J. Stevens, T. Mukai, D. Ohnishi, R.A. Hogg, "Non-destructive mapping of doping and structural composition of MOVPE-grown high current density resonant tunnelling diodes through photoluminescence spectroscopy”, Journal of Crystal Growth, vol. 418, 102 (2015)

[11] M. Grundmann, D. Bimberg, "Anisotropy elects on excitonic properties in realistic quantum wells" Phys. Rev. B, vol.35, no.18, pp.13486-13489 (1988)

[12] M. A. Herman, D. Bimberg, J. Christen, "Heterointerfaces in quantum wells and epitaxial growth processes: Evaluation by luminescence techniques", Journal of Applied Physics, vol. 70, R1 (1991)

[13] K.J.P. Jacobs, B.J. Stevens, R.A. Hogg (in press), "Photoluminescence Characterisation of High Current Density Resonant Tunnelling Diodes for Terahertz Applications," IEICE Transactions.

[14] N.N. Ledentsov, J. Bhrer, M. Beer, F. Heinrichsdorff, M. Grundmann, D. Bimberg, S.V. Ivanov, B.Ya. Meltser, S.V. Shaposhnikov, I.N. Yassievich, P.S. Kopev, Z.I. Alferov, “Radiative states in type-II GaSb/GaAs quantum wells” „Phys. Rev., B 52 (1995), 14058.

[15] I. Vurgaftman, J. R. Meyer, and L. R. Ram-Mohan, “Band parameters for III-V compound semiconductors and their alloys” , Journal of Applied Physics, vol. 89, 5815 (2001) 
